\title{
The Fraction of Cortical GABAergic Neurons Is Constant from Near the Start of Cortical Neurogenesis to Adulthood
}

\author{
Setsuko Sahara, ${ }^{1}$ Yuchio Yanagawa, ${ }^{2}$ Dennis D. M. 0’Leary, ${ }^{1}$ and Charles F. Stevens ${ }^{1}$ \\ ${ }^{1}$ Molecular Neurobiology Laboratory, The Salk Institute, La Jolla, California 92037, and ${ }^{2}$ Department of Genetic and Behavioral Neuroscience, Gunma \\ University Graduate School of Medicine, Maebashi 371-8511, Japan
}

\begin{abstract}
Approximately one in five neurons is GABAergic in many neocortical areas and species, forming a critical balance between inhibition and excitation in adult circuits. During development, cortical GABAergic neurons are generated in ventral telencephalon and migrate up to developing cortex where the excitatory glutamatergic neurons are born. We ask here: when during development is the adult GABAergic/ glutamatergic neuron ratio first established? To answer this question, we have determined the fraction of all neocortical GABAergic neurons that will become inhibitory $\left(\mathrm{GAD}{ }^{+}\right)$in mice from embryonic day 10.5 (E10.5) to postnatal day $28(\mathrm{P} 28)$. We find that this fraction is close to $1 / 5$, the adult value, starting from early in corticogenesis (E14.5, when GAD67 ${ }^{+}$neurons are still migrating tangentially to the cortex) and continuing at the same $1 / 5$ value throughout the remainder of brain development. Thus our data indicate the one-in-five fraction of GABAergic neurons is already established during their neuronal migration and well before significant synapse formation.
\end{abstract}

\section{Introduction}

Across many cortical regions and species, approximately one in five neurons in the adult neocortex is inhibitory and uses the neurotransmitter GABA to hyperpolarize its target neurons (Hendry et al., 1987). Cortical GABAergic inhibitory neurons come in a rich variety of types, and the numbers and cortical locations of each type are believed to be tightly regulated in cortical neural circuits (Markram et al., 2004; Huang et al., 2007; Ascoli et al., 2008).

Setting the fraction of all neurons that are GABAergic at $1 / 5$ as seen in the adult cortex is more complicated than it might first seem. During development, glutamatergic and GABAergic neurons are born in separate locations. The glutamatergic neurons are generated in the ventricular and subventricular zones of embryonic cortex and typically migrate radially in an "inside-out" sequence to form layers in the cortical plate (O'Leary et al., 2007; Marín et al., 2010). Most, if not all, GABAergic neurons, however, are born in the medial and caudal ganglionic eminences in ventral telencephalon, and migrate first tangentially from their birthplace to the proper cortical region, and then radially through the cortical plate to reach their final laminar location (Anderson et al., 1997; Lavdas et al., 1999; Nery et al., 2002). The tangential

Received Dec. 22, 2011; revised Feb. 2, 2012; accepted Feb. 12, 2012.

Author contributions: S.S. and C.F.S. designed research; S.S. and C.F.S. performed research; Y.Y. contributed unpublished reagents/analytic tools; S.S., D.D.M.O., and C.F.S. analyzed data; S.S., D.D.M.O., and C.F.S. wrote the paper.

This work was supported by NIH Grants R37 NS31558 and R01 MH086147 (D.D.M.O.). D.D.M.O. and C.F.S. are supported by the Vincent J. Coates Chair in Molecular Neurobiology. We thank Edward Callaway for materials and Hiroki Taniguch and Josh Huang for sharing unpublished observations.

Correspondence should be addressed to Dennis D. M. O'Leary, Molecular Neurobiology Laboratory, The Salk Institute, 10010 N. Torrey Pines Road, La Jolla, CA 92037. E-mail: doleary@salk.edu.

S. Sahara's present address: MRC Centre for Developmental Neurobiology, Kings College London, New Hunts House, Guys Campus, London SE1 1UL, UK.

DOI:10.1523/JNEUROSCI.6412-11.2012

Copyright $\odot 2012$ the authors $\quad 0270-6474 / 12 / 324755-07 \$ 15.00 / 0$ migration of these cells mainly follows two distinct streams, one superficial in the marginal zone and a deeper one in the subventricular and intermediate zones (Ang et al., 2003; Kriegstein and Noctor, 2004; Tanaka et al., 2006; Marín et al., 2010). After cells have reached their appropriate tangential location in the cortex, they move radially — perhaps after a considerable delay — to reach their final laminar position in the cortical plate; on occasion, GABAergic cells take more complicated paths to their final location (Tanaka et al., 2006).

Many GABAergic neurons seek out inside-out locations populated by excitatory neurons born at the same time (Fairén et al., 1986; Cavanagh and Parnavelas, 1988; Ang et al., 2003; Valcanis and Tan, 2003; Xu et al., 2004; Yozu et al., 2004; Rymar and Sadikot, 2007) and some seem to recognize layer-specific cues provided by excitatory neurons for settling their final positions (Lodato et al., 2011). But others do not follow this rule so that they are not birthdate-matched with their neighboring excitatory cells (Yozu et al., 2004; Rymar and Sadikot, 2007). Thus, the GABAergic/glutamatergic neuron number matching must be achieved in the context of the spatially and temporally dispersed neuron generation with superimposed cellular migration over different routes and at different times.

What are the developmental mechanisms responsible for setting the fraction of cortical GABAergic neuron at one in five? Are excess GABAergic neurons provided to the cortex and their final number adjusted in respond to the environment cues as observed in some developmental processes (Cowan et al., 1984; Kuan et al., $2000)$ ? Or is the appropriate quantity of GABAergic neurons determined by the number generated remotely and by migratory choices? A first step in approaching this question is to determine when during development this $1 / 5$ ratio is established. We have estimated the GABAergic neuron fraction in the developing neocortex on each embryonic day from embryonic day 10.5 (E10.5) through E18.5 and at several times after birth, and have found 
that this fraction is set at close to $1 / 5$ early in corticogenesis. Nearly the same fraction is then maintained throughout the remainder of development and into adulthood.

\section{Materials and Methods}

Animals. Timed pregnant ICR mice and GAD67-GFP knock-in mice (Tamamaki et al., 2003) were used in accordance with institution guidelines. We crossed heterozygote males to wild-type ICR to obtain embryos and pups at the ages described. The day of insemination and the day of birth are designated as embryonic day 0.5 (E0.5) and postnatal day 0 $(\mathrm{P} 0)$, respectively.

Histochemistry. For counting GAD67-GFP cells on sections, brains were fixed in $4 \%$ PFA in PBS, cryoprotected in $20 \%$ sucrose/PBS, cut at $20 \mu \mathrm{m}$. For identifying neurons at the later stages (P7 and P28), sections $(40 \mu \mathrm{m})$ were stained by mouse anti-NeuN antibody (Millipore Bioscience Research Reagents). Sections were counterstained by TO-PRO-3 iodide (Invitrogen) and observed with confocal microscopy (Zeiss LSM510). GFP-positive, NeuN-positive, and total cells were counted under $50 \mu \mathrm{m}$ columns in the developing somatosensory areas $(n=4)$. For immunostaining with anti-GABA antibody, brains of embryonic or postnatal GAD67-GFP mice were fixed in $0.2 \%$ glutaraldehyde- $4 \%$ PFA in $0.1 \mathrm{~m}$ phosphate buffer, $\mathrm{pH} 7.4$, overnight. Brain sections $5 \mu \mathrm{m}$ thick were stained with rabbit anti-GABA antibody (Sigma) and visualized with Cy3 anti-rabbit secondary antibody. Cell death was assessed by the staining of E14.5-E18.5 coronal sections from GAD67-GFP heterozygote mice $(n=5)$ by rabbit anti-activated caspase- 3 antibody (Millipore Bioscience Research Reagents). For double staining of NeuN and Synapsin I, P7 and P28 sections (10 pictures for each taken randomly from 5 brains) were stained by in situ hybridization with Synapsin I probes (Allen brain atlas: RP_050927_04_H08) according to the manufacturer's fluorescent in situ protocol (TSA kit with HRP biotin tyramide, Life Technologies) and subsequently subjected to immunostaining with anti-NeuN antibody.

Dissociated cells were prepared by dissecting out cortices from 8-14 embryos at each of the indicated stages as described earlier (Sahara and O'Leary, 2009) but with some modification. Briefly, the middle $1 / 9$ part of developing cortex is dissected out (with the remaining rostral, caudal, medial, and lateral regions discarded), incubated in $0.2 \%$ trypsin, $1 \mathrm{~mm}$ cysteine, $7 \mathrm{U} / \mathrm{ml}$ papain, $0.5 \mathrm{mg} / \mathrm{ml}$ collagenase (for E16.5-E18.5) in HBBS for $10 \mathrm{~min}$. Cells are dissociated by pipetting on ice and are immediately fixed in $4 \%$ PFA and attached poly-L-lysine cortex cover glasses by centrifugation. Cells are immunostained by mouse anti-TuJ1 (Covance) and rabbit anti-Map2 (Millipore Bioscience Research Reagents) antibodies. Ten to 15 pictures are taken from 3-4 independent experiment sets prepared typically from 2-3 embryos for counting of dissociated cells, and an average of $\sim 1700$ total cells were counted per stage (range 5003000 , depending on the age).

\section{Results}

\section{Faithful expression of GAD67-GFP cells in GABA ${ }^{+}$ cortical cells}

To count cortical GABAergic neurons during development, we used heterozygotic GAD67-GFP knock-in mice in which the expression of GAD67 is marked by eGFP (Tamamaki et al., 2003). This approach is necessary because immunostaining of GABA ${ }^{+}$ cells by anti-GABA antibody was not reproducible in dissociated cells (data not shown), although the antibody gives reliable staining in thin sections fixed with $0.2 \%$ glutaraldehyde. Both GAD65 and 67, the two main GAD isoforms, are believed to be expressed in the same GABAergic neurons in the cortex. GAD67-GFP mice are therefore expected to have nearly all cortical GABAergic interneurons labeled (Esclapez et al., 1993, 1994). Also in this mouse, $>96 \%$ of $\mathrm{GABA}^{+}$-immunoreactive neurons have been reported to express GFP at E13.5 and E15.5 (Tanaka et al., 2006). We have examined coexpression of GABA and GAD67-GFP at other embryonic and postnatal stages and find that GAD67-GFP is expressed by $>93 \%$ of $\mathrm{GABA}^{+}$neurons at all stages examined
(Fig. 1A-P). This observation confirms that GAD67-GFP is a faithful reporter for GABA-expressing cells.

Brains from heterozygous GAD67-GFP neonates contain only $\sim 60 \%$ of the total quantities of GABA compared with wild-type mice (Tamamaki et al., 2003), and we worried that this GABA reduction might affect the migratory behavior of GABAergic neurons as ambient GABA has been suggested to promote cortical invasion of GABAergic neurons into the cortex (Cuzon et al., 2006). After immunostaining with anti-GABA antibody, however, we find indistinguishable migratory patterns (Fig. 1Q-T) and GABAergic neuron numbers between wild-type and heterozygote (Fig. 1U). We therefore believe that conclusions derived from the study of $\mathrm{GFP}^{+}$cells are applicable to wild-type cortex.

\section{Migration profiles of GAD67 ${ }^{+}$neurons in the cortex}

The first step in our analysis was to examine the spatial distribution of $\mathrm{GAD} 7^{+}$cells during development. We find that no $\mathrm{GAD} 7^{+}$cells have entered the cortex by E12.5 (data not shown), and that the wavefront of migrating GABAergic cells arrives in cortex from the ganglionic eminences by E13.5 (Fig. $2 A, A^{\prime}$ ). Most $\mathrm{GAD} 7^{+}$neurons generated in the ganglionic eminences continue to stream tangentially into the cortex through the marginal zone and the subventricular/intermediate zones (the superficial and deep migratory streams, respectively), although a small number of GAD $67^{+}$cells may take alternative routes (Fig. $2 B-G$, $B^{\prime}-G^{\prime}$ ) (Nadarajah et al., 2002). Some time after following their tangential course to the proper location, GABAergic neurons migrate radially to their final laminar locations in the cortical plate (Fig. $2 F-I, F^{\prime}-I^{\prime}$ ).

We have also quantified the migration of cortical GABAergic neurons generated in the ganglionic eminences by counting on sections the fraction of all GAD $67^{+}$cells that are present in each of three zones: (1) the cortical plate (including cells in the upper intermediate zone) where nearly all GABAergic neurons ultimately reside, (2) the superficial migratory stream (marginal zone) and (3) the deep migratory stream (subventricular zone/ intermediate zone including those cells in ventricular zone) (Fig. $2 J)$. By P0, a time when most of the excitatory neurons have moved into the cortical plate and many have reached their assigned laminar positions, approximately half of the GABAergic neurons remain outside the cortical plate and are approximately equally divided between the two migratory streams.

\section{Estimation of the ratio of cortical GABAergic neurons during development}

Our main goal in this paper is to determine, at each stage of embryonic and early postnatal development, what fraction of cortical neurons are GABAergic. This is accomplished in three steps. The first step is to estimate, at each age, what fraction of all cortical cells are neurons, and the second step is to find what fraction of all cortical cells are $\mathrm{GAD} 7^{+}$. In the final step we divide the second fraction by the first to find the fraction of all neurons that are $\mathrm{GAD} 67^{+}$at the various ages.

\section{Fraction of all cortical cells that are neurons}

Early in neurogenesis, the vast majority of cells present in the developing cortex are progenitors, but by $\mathrm{P} 0$, progenitors account for only a small fraction of all cortical cells, with neurons now being in the majority. We have determined, from E10.5 through P28, what fraction of all cells present in the developing neocortex is neurons. Before developmental age $\mathrm{P} 0$, cortical neurons are stained with well established early and late neural marker antibodies, TuJ1, and with Map2 (Ferreira et al., 1987; Ferreira 

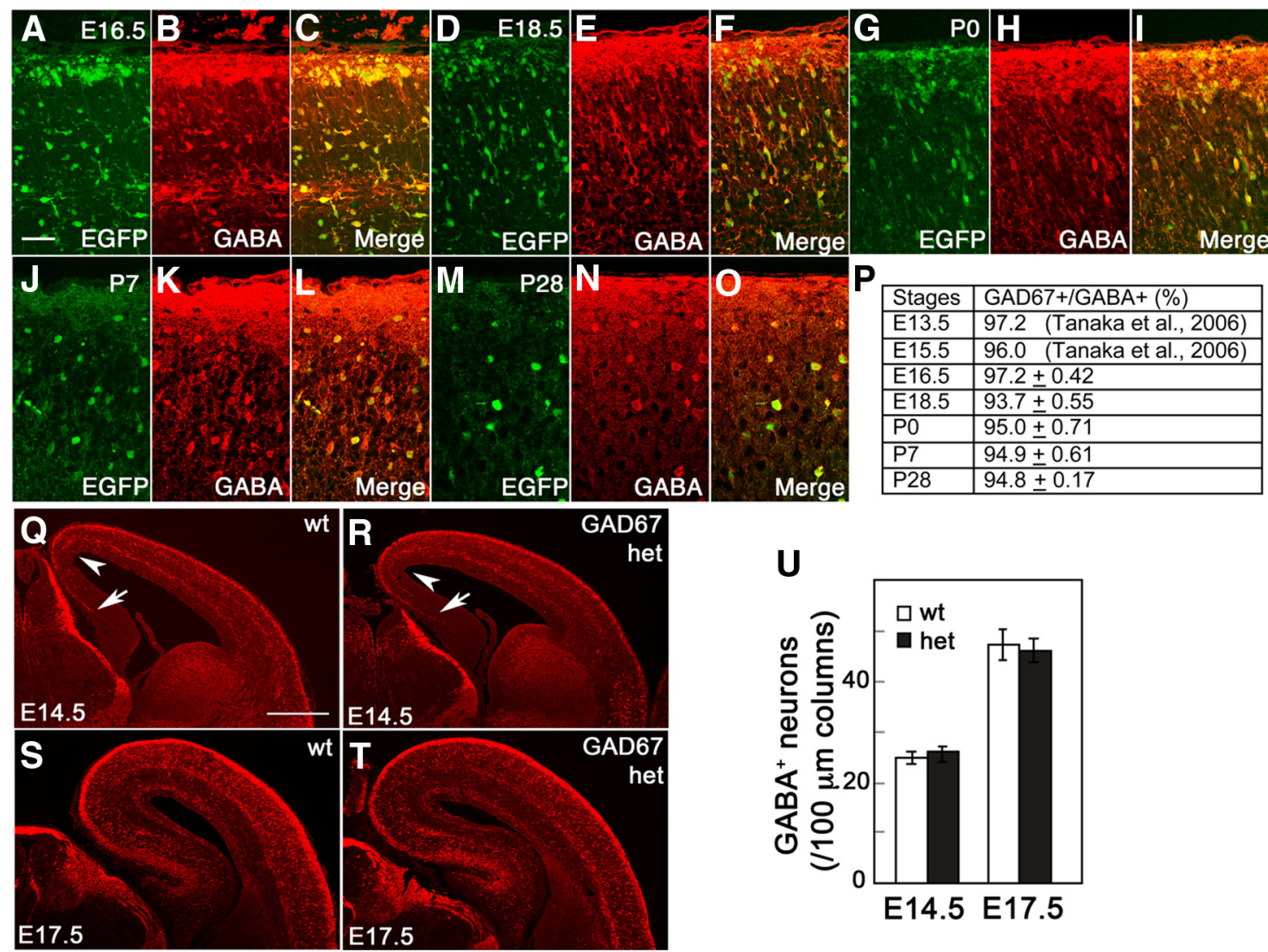

Figure 1. Faithful expression of GAD67-GFP in cortical GABAergic neurons. A-P, Immunostaining of GAD67 ${ }^{+}$brain sections with GABA antibody at the stages indicated. GAD67-GFP signals $(\boldsymbol{A}, \boldsymbol{D}, \boldsymbol{G}, \boldsymbol{J}, \boldsymbol{M})$ were colocalized with those of $\mathrm{GABA}(\boldsymbol{B}, \boldsymbol{E}, \boldsymbol{H}, \boldsymbol{K}, \boldsymbol{N}$, single images; $\boldsymbol{C}, \boldsymbol{F}, \boldsymbol{I}, \boldsymbol{L}, \boldsymbol{O}$, merged images). More than $93 \%$ of $G A D 67-G F P$ cells are immunopositive for $G A B A$ at the stages examined $(\boldsymbol{P}, n=4$, SEM as indicated). $\boldsymbol{Q}-\boldsymbol{U}$, Similar migratory patterns of GABAergic neurons in heterozygous GAD67-GFP and wild-type cortex. Sections of E14.5 ( $\boldsymbol{Q}, \boldsymbol{R})$ and E17.5 embryonic brains $(\boldsymbol{S}, \boldsymbol{T})$ of wild-type $(\boldsymbol{Q}, \mathbf{S})$ and GAD67-GFP het $(\boldsymbol{R}, \boldsymbol{T})$ mice are stained with anti-GABA antibody. Overall, migratory patterns appear to be the same in both brains at both stages. GABA ${ }^{+}$cells in each section have also been counted in the somatosensory cortex $(\boldsymbol{U})$, and no significant differences in the numbers of $\mathrm{GABA}^{+}$cells are found between genotypes. Scale bars: (in $\mathbf{A}) \boldsymbol{A}-\mathbf{0}, 50 \mu \mathrm{m}$; (in Q) $\mathbf{Q}-\boldsymbol{T}, 0.5 \mathrm{~mm}$.

and Caceres, 1992; Menezes and Luskin, 1994). Because the antigens recognized by these antibodies are cytoskeletal proteins, it is difficult to count accurately all of the $\mathrm{TuJ} 1^{+}$and Map $2^{+}$cells present in sections because closely packed cells obscure one another. Thus we followed the strategy of preparing dissociated cells to count cell numbers at each of the developmental stages (Sahara and O'Leary, 2009). We dissected out the developing neocortical tissue of GAD67 heterozygote embryos for each day from E10.5 to E18.5, and dissociated and plated cortical cells on a glass coverslip with immediate fixation for acute immunostaining and counting (Fig. $3 A-G$ ). Cells are DAPI stained so that we can count all cells present (Fig. $3 A$ ), and then the fractions of cells that express TuJ1 (Fig. $3 B, D$ ) and/or Map2 (Fig. 3C,D) are determined.

Very few neurons are present at E10.5 (2.8\% of all cells) most cells are progenitors at this age-and newly born cortical neurons begin to appear in appreciable numbers on E12.5 $(\sim 17 \%$ of all cells). The fraction of cells that are neurons (calculated as neurons per total cells) increases steadily with embryonic age and reaches a little $<50 \%$ by E14.5 (Fig. $4 A$ ) and nearly $90 \%$ by E18.5 as progenitors undergo terminal divisions and are replaced by neurons and glial progenitors. The fraction of all cortical cells that are neurons is presented, as a function of age, in Figure $4 A$ (circles).

By P7, neurons become positive for immunostaining by another neuronal marker, the nuclear antigen NeuN (Mullen et al.,
1992), which allows us to count stained nuclei on sections and simultaneously to observe the location of $\mathrm{GAD} 67^{+}$cells (see below). We counted the fraction of TO-PRO3 cells that are $\mathrm{NeuN}^{+}$ in sections of cortex at ages P7 and P28 (Fig. $3 H-K$ ) because dissociation is difficult at these later stages when neural circuits are forming and maturing. Note that we omitted the neuron counts for P0 because weak NeuN immunostaining does not provide an accurate label for all neurons at this age. Because of glial cell generation, the fraction of cells that are neurons declines at P28 (Fig. 4A).

Although NeuN has been widely used for as a general neuronal marker, we are not aware of a quantitative analysis that shows NeuN expression marks all cortical neurons. Thus we performed double labeling of NeuN and Synapsin I, a highly reliable marker for neurons (Micheva et al., 2010). Although the Synapsis I protein is localized at synapses, we took advantage of the fact that Synapsin I mRNA can be detected surrounding neuronal nuclei (Fig. $3 L-O$ ). When we counted cells coexpressing NeuN and Synapsin I, we found that $\sim 97 \%$ of the NeuN + cells are positive for Synapsin I (97.2\% \pm 0.62 SEM for P7, 97.3\% \pm 0.78 SEM at P28). We therefore conclude that NeuN is a faithful marker of cortical postnatal neurons under the conditions of our experiments.

\section{Fraction of all cortical cells that are GAD67 ${ }^{+}$}

Next we counted the numbers of GAD67 ${ }^{+}$cells prepared from GAD67 knock-in mice (Fig. $3 E-K$ ). Since the counting of all 

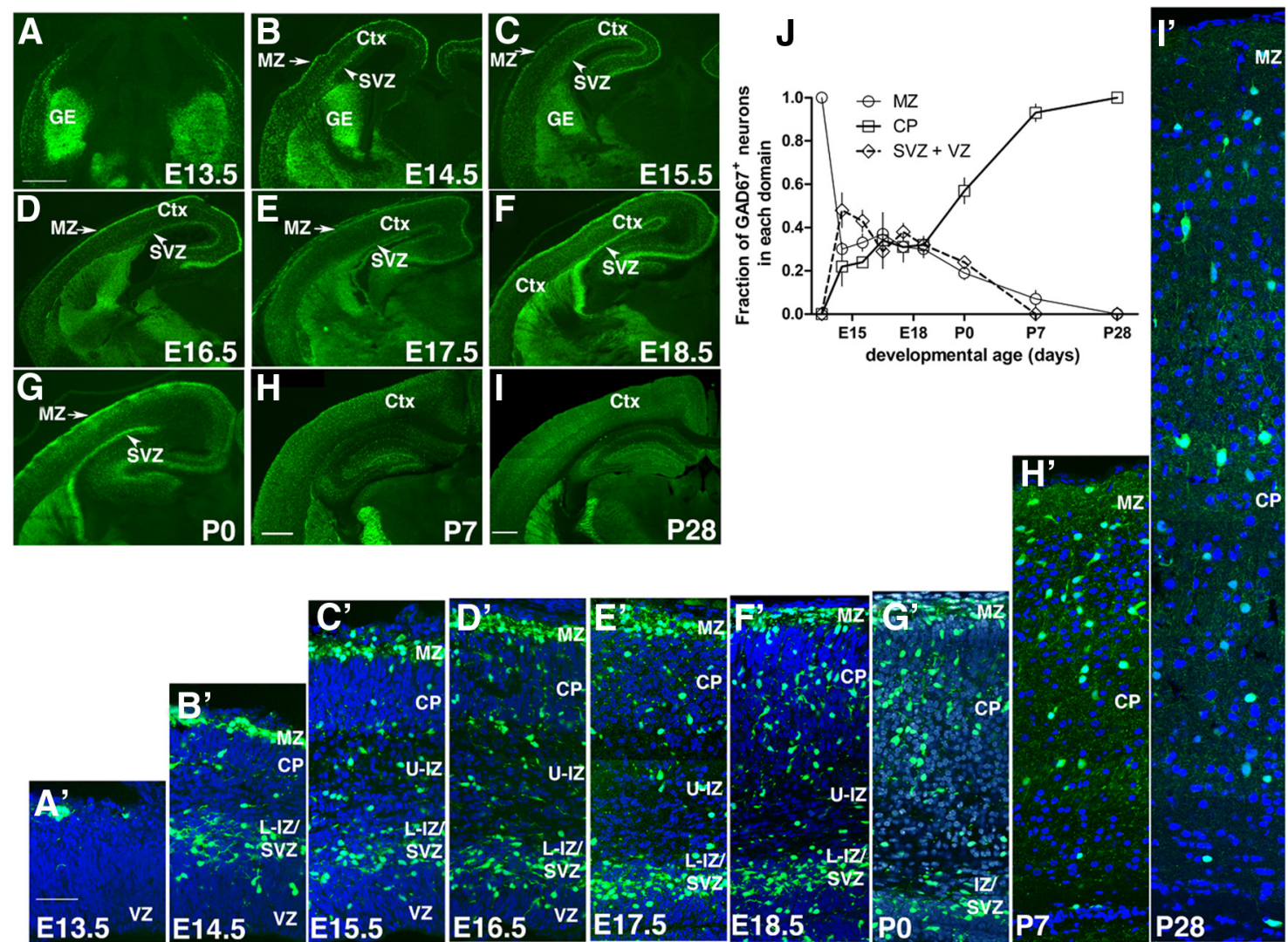

Figure 2. Migration patterns of GAD67-GFP cells during development. $A-I$, Images of GAD67-GFP heterozygote mouse brains at indicated stages. $\boldsymbol{A}^{\prime}-\boldsymbol{I}^{\prime}$, High-magnification pictures of $\boldsymbol{A}-\boldsymbol{I}$ at middle sections along the anterior/posterior and lateral/medial axes. GAD67 ${ }^{+}$cells begin to reach to the cortex tangentially at E13.5 $\left(\boldsymbol{A}, \boldsymbol{A}^{\prime}\right)$ over the two main migratory streams in the marginal zone (MZ) and subventricular zone (SVZ), and fill in those pathways at E14.5 (B, $\left.\boldsymbol{B}^{\prime}\right)$. Some cells enter the cortical plate (CP) and upper/lower intermediate zone (U-IZ and L-IZ) and migrate in these regions, but most cells use tangential streams $\left(\boldsymbol{C}-\mathbf{G}, \boldsymbol{C}^{\prime}-\mathbf{G}^{\prime}\right)$. Entry into the cortical plate by $G A D 67{ }^{+}$cells becomes evident around E17.5-E18.5 $\left(\boldsymbol{E}^{\prime}, \boldsymbol{F}^{\prime}\right)$ and most of these GABAergic cells have settled to their final position by P28 $\left(\boldsymbol{H}, \boldsymbol{I}, \boldsymbol{H}^{\prime}, \boldsymbol{I}^{\prime}\right)$.J, Distribution of GAD67 ${ }^{+}$cells that are found in each of three cortical domains for each embryonic day. Ordinate is fraction of all GAD67 ${ }^{+}$cells (error bars indicate SEM) in marginal zone (MZ, open circles), in the cortical plate (CP, open squares), and in the lower subventricular zone and ventricular zone (SVZ + VZ, open diamonds), and abscissa is developmental age (days). Scale bars: (in $\boldsymbol{A}) \boldsymbol{A}-\mathbf{G}, 0.5 \mathrm{~mm} ; \boldsymbol{H}, 0.5 \mathrm{~mm} ; \boldsymbol{I}, 0.5 \mathrm{~mm}$; (in $\boldsymbol{A}^{\prime}$ ) $\boldsymbol{A}^{\prime}-\boldsymbol{I}^{\prime}, 50 \mu \mathrm{m}$.

cortical neurons at embryonic stages must be performed with dissociated cells, we determined the number of GAD $67^{+}$cells in the same way. To determine whether the results of counting GAD67 ${ }^{+}$markers with dissociated cells differs from counting in sections, we used both methods over a range of developmental stages, and found the two methods agree (data not shown). The GAD $67^{+}$cells at postnatal stages are counted in sections to compare with numbers of $\mathrm{NeuN}^{+}$cells. We find that the fraction of GABAergic cells increases steadily from E14.5 through the rest of embryonic development, plateaus at $\mathrm{P} 0$, and then declines slightly at P28, again presumably because of the postnatal generation on non-neuronal cells (glia) (Fig. 4A).

Fraction of all cortical neurons that are GAD67 $7^{+}$

Above we have determined what fraction of all cells are neurons, and what fraction of all cells are GAD67 ${ }^{+}$(Fig. 4A). The ratio of these two fractions tells us what fraction of neurons are GABAergic. We have plotted this quantity for each day from E12.5 through E18.5 and for P7 and P28 in Figure 4B. No GAD67 ${ }^{+}$ neurons are present in the cortex at E12.5, although almost 20\% of all cells present are neurons. The wave of migrating GAD67 ${ }^{+}$ cells from the ganglionic eminences starts arriving at E13.5 and only $\sim 3 / 100$ of the neurons are GABAergic on that day. But by the next day (E14.5), $\sim 1 / 5$ of the neurons in cortex are GAD67 ${ }^{+}$ (Fig. $3 B$ ), even though most GABAergic neurons present in the developing cortex (approximately three quarters) have not yet entered the cortical plate (Fig. $2 B^{\prime}$ ). This $1 / 5$ GABAergic cell fraction then is maintained almost unchanged throughout the remainder of development, despite the fact that not all GABAergic neurons have reached their assigned laminar position until after P7, and they are still excitatory (see Discussion).

We conclude, then, that the adult fraction of GABAergic $\sim 20 \%$ - is reached on E14.5 when at least $80 \%$ of cortical interneurons are still in their migratory mode, and this same 1/5 fraction is then maintained throughout development and for the remainder of the animal's life.

\section{Discussion}

Possible mechanisms for generating and maintaining the $1 / 5$ GABAergic neuron fraction

We have shown here that the $1 / 5$ fraction of cortical GABAergic neurons is set up early in corticogenesis when GABAergic neurons are still in their migratory mode. How might this occur? For the matching of GABAergic/glutamatergic neuron numbers, some combination of at least three mechanisms could be involved: cell death, regulation of neuronal generation rates, and control of GABAergic neuronal migration.

If cell death is important, perhaps excess neurons, either glutamatergic or GABAergic, die during development in numbers necessary to establish and maintain the $1 / 5$ GABAergic neuron fraction. We find that cell death of both glutamatergic and 

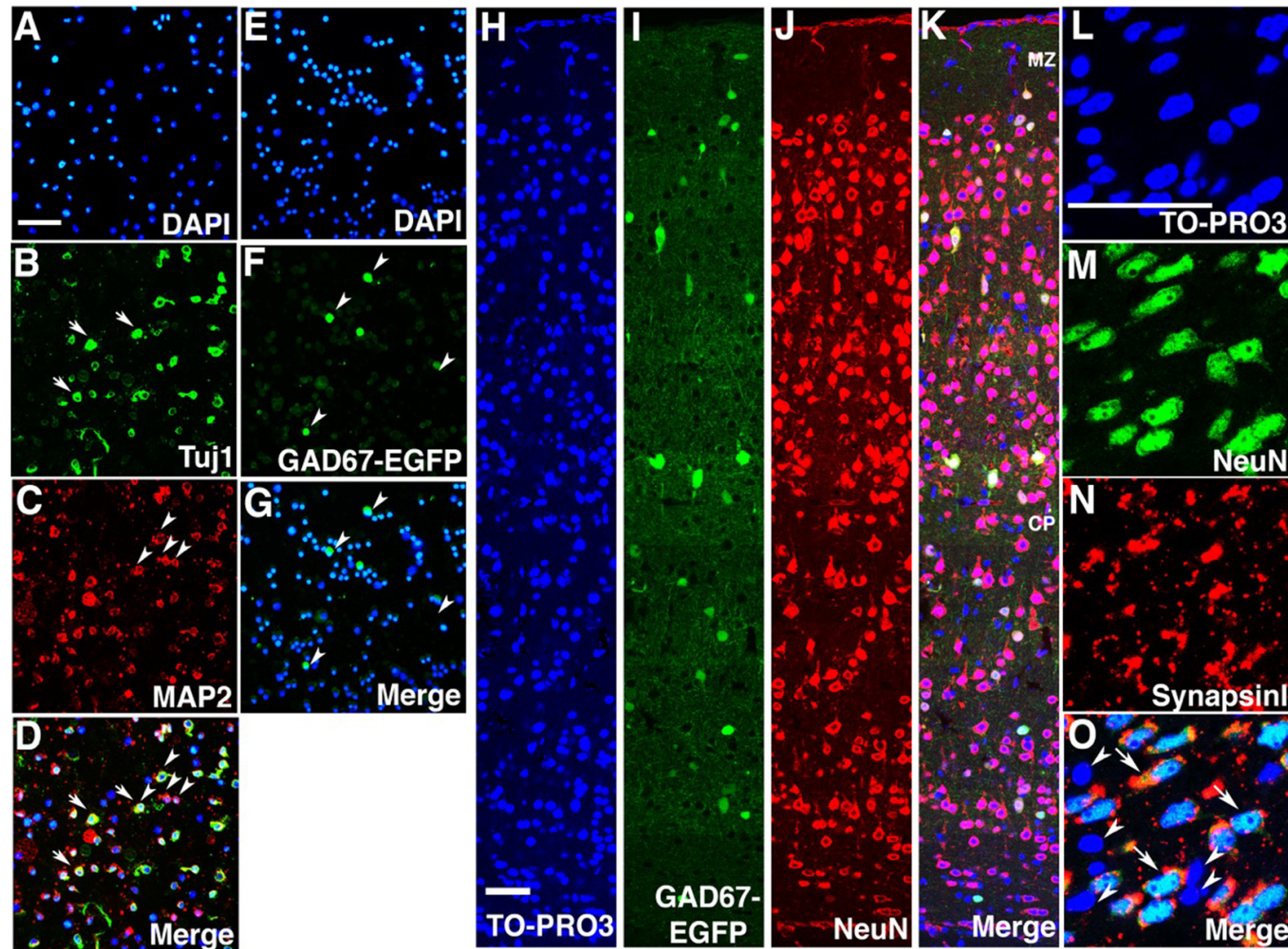

Figure 3. Cortical cells stained with neuronal markers. $\boldsymbol{A}-\mathbf{G}$, Dissociated cells from embryonic mouse cortex at various stages from E10.5 to E18.5 are stained on a glass coverslip by TuJ1 ( $\boldsymbol{B}$ ) and anti-Map2 ( () antibodies. Representative images at E14.5 are shown. GFP signals are detected in dissociated cells prepared from GAD67-GFP mice $(\boldsymbol{F})$. Merged images are shown in $\boldsymbol{D}$ and $\boldsymbol{G}$. Cells are counterstained with DAPI $(\boldsymbol{A}, \boldsymbol{E})$ to label all cortical cells. Typical images of an E14.5 cortex are shown. Arrows indicate Tuj $1^{+}$cells and arrowheads Map2 ${ }^{+}$cells $(\boldsymbol{A}-\boldsymbol{D})$, or GAD67-GFP cells $(\boldsymbol{F}$, G). Scale bar: (in $\boldsymbol{A}) \boldsymbol{A}-\mathbf{G}, 0.2 \mathrm{~mm}$. $\boldsymbol{H}-\boldsymbol{K}$, Sections of GAD67-GFP postnatal brains at P28 were stained by NeuN antibodies $(\boldsymbol{J})$. GFP signals indicate GAD67 ${ }^{+}$cells $(\boldsymbol{I})$ that have been counterstained with TO-PR03 to visualize all cell nuclei. Merged image in $\boldsymbol{K}$. Scale bar: (in $\boldsymbol{H}) \boldsymbol{H}-\boldsymbol{K}, 50 \mu \mathrm{m}$. $\boldsymbol{L}-\mathbf{0}$, Faithful expression of NeuN in the cortical neurons. P28 sections after fluorescence in situ hybridization $(\boldsymbol{N})$ and subsequent immunostaining with anti-NeuN antibody $(\boldsymbol{M})$. Sections were counterstained with TOP03 and the merged image is shown in $\mathbf{0}$. Most, if not all, of NeuN ${ }^{+}$cells are colocalized with Synapsin I mRNA. Arrows indicate double-positive cells for NeuN and Synapsin I and arrowheads negative for both (0), Scale bar: (in $\boldsymbol{L}$ ) $\boldsymbol{L}-\mathbf{0}, 50 \mu \mathrm{m}$.
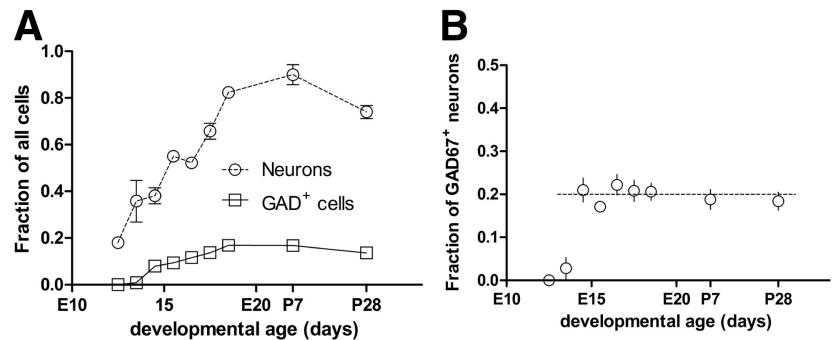

Figure 4. A, Fraction of all cells that are neurons and are GABAergic neurons during embryonic development and in the early postnatal period. Ordinate is the fraction of all neurons (open circles) and GAD67 ${ }^{+}$neurons (open squares) relative to all cells present in cortex, and the abscissa is developmental age (days). $\boldsymbol{B}$, Fraction of all neurons that are $\mathrm{GAD}^{+}{ }^{+}$for each developmental age. Ordinate is fraction of neurons that are $\mathrm{GAD} 7^{+}$and abscissa is developmental age (days). The dotted line is the fraction $1 / 5$. Dissociated cells are counted from 3-4 independent experiments. Error bars indicate SEM.

GABAergic neurons during embryonic stages is much too rare to account for significant adjustments in neuron numbers (data not shown). However, at postnatal stages, a subtype-specific reduction of GABAergic neurons occurs, presumably due to cell death (Taniguchi $\mathrm{H}$ and Huang J, personal communication). This cell type-specific cell death could contribute to, and perhaps explain, the slight decline in the GABAergic/glutamatergic neuron fraction seen in Figure $4 B$ at postnatal times. Further evidence against extensive cell death as a regulatory mechanism to control the fraction of GABAergic cells is provided by the observations of Southwell et al. (2010) who found that at least some embryonic GABAergic cells transplanted into the visual cortex of mice during the first two postnatal weeks survived and functioned.

Cortical GABAergic/inhibitory and glutamatergic/excitatory neurons are born in distinct domains. Thus, if proliferation of GABAergic neurons is coordinated with the birth of glutamatergic neurons, some feedback mechanisms between cortical glutamatergic and GABAergic neurons are used to achieve the correct balance of neuron numbers (Lander et al., 2009). For instance, Emx1 and Emx 2 are expressed in cortical progenitors but not in the ventral progenitors; double Emx1/Emx2 knock-out mice are known to have a smaller neocortex, although there appears to be an increase in the volume of the ganglionic eminences at E13.5 (Shinozaki et al., 2002, their Fig. 1G,E). Thus it should be informative to examine proliferation of cortical GABAergic neurons in mice whose cortical size is changed.

Regulation of GABAergic neuron migration must be somehow involved in maintaining a constant GABAergic/glutamatergic neuron ratio; failure of proper migration, for example, would mean that too few $\mathrm{GAD} 7^{+}$cells would be present in cortex even if correct numbers of cells were generated. Tangential migration of cortical GABAergic interneurons is known to be regulated by the combined actions of diverse signals (Marín et al., 2010). Some of these secreted factors, like CXCL12 and neuregulins, are ex- 
pressed in cortical progenitors and influence the migrating interneurons resulting in the different fraction of GABAergic neurons (Flames et al., 2004; López-Bendito et al., 2008; Li et al., 2012).

A factor that has been identified as a regulator of GABAergic neuron migration is the concentration of GABA itself (Cuzon et al., 2006). Furthermore, the expression of KCC2 - which is responsible for the developmental switch in the response of neurons to GABA from depolarizing to hyperpolarizing (Ben-Ari, 2002) — also appears to be involved in promoting or inhibiting migration (Bortone and Polleux, 2009). These observations mean that GABAergic neurons may, at least in part, regulate their own migration and thus help to control the GABAergic/total neuron ratio.

This possibility of GABAergic neuronal self-regulation of migration, however, presents us with a puzzle. On the one hand, we find that migration to cortex from the ganglionic eminences is unaffected in GAD67-GFP mice but, on the other hand, these mice have been reported to have GABA content reduced to $60 \%$ of that found in wild-type mice (Tamamaki et al., 2003). If neuronal migration is influenced by GABA concentration, why do we find migration unaffected in mutant mice with reduced GABA levels? Two obvious alternatives present themselves. First, the effects of GABA on migration presumably depend on released ambient GABA, and the relation between GABA release and the decreased GABA content of GABAergic neurons during development is unknown. A likely alternative, then, is that despite a $40 \%$ reduction in overall GABA content, the concentration of GABA present at the relevant times and locations to affect migration is not sufficiently changed in the GAD67-GFP mice to alter migration. A more complex alternative is that the dependence of GABAergic neuron migration on GABA concentrations is under homeostatic regulation. For example, decreased GABA concentrations might inhibit migration directly but might simultaneously, through decreasing the expression of KCC2 (Ganguly et al., 2001) or other regulators and thereby slowing the conversion of GABAergic neurons from excitatory to inhibitory, facilitate migration in a way that leaves net migration unaffected. These and other possibilities will require further experiments to evaluate. Together with published observations and our findings, the $1 / 5$ ratio of cortical GABAergic/glutamatergic neurons must be controlled by a number of factors involved in generation and migration of GABAergic neurons.

\section{Are there subtype-specific feedback pathways to keep GABAergic/glutamatergic ratio constant?}

In this study, we treated GABAergic neurons as if they constituted a uniform population and found that the GABAergic /glutamatergic neuronal ratio is constant during development. We know, however, that GABAergic neurons come in different subtypes with distinct origins, morphologies, and functions. Furthermore recent papers indicate that the laminar position of various cortical interneuron subtypes is regulated either by activity or by the presence of excitatory neurons in the different layers (De Marco Garcia et al., 2011; Lodato et al., 2011). Although the total population size of GABAergic neurons is fixed relative to the number of excitatory neurons, region-to-region and layer-to-layer differences exist for particular GABAergic neuron subtypes, and understanding these differences is an important aspect of knowing the meaning of the fixed 1/5 ratio, both during development at in the adult brain.

\section{Functional influence of developmental and exogenous perturbation of GABAergic/glutamatergic ratio}

Clinically the loss of cortical inhibitory neurons is thought to be involved in various brain disorders such as schizophrenia and epilepsy (Huang et al., 2007; Ascoli et al., 2008). Recent advances in the generation of particular neuronal types from ES/iPS cells offer a potential route to treating these diseases through the transplantation of supplemental GABAergic neurons into adult brains in which neurogenesis is no longer taking place (Kriegstein and Pitkänen, 2009). Such a transplantation strategy will not be able to introduce precise numbers of neurons into specific regions of the intact brain. Implementing this type of treatment strategy, therefore, depends on knowing the numbers of inhibitory subtypes present in various locations, on understanding how their number is regulated, and on learning the consequences of incorrect numbers. Refinements of the approach we have taken here constitutes, we believe, an important step in this direction.

\section{References}

Anderson SA, Eisenstat DD, Shi L, Rubenstein JL (1997) Interneuron migration from basal forebrain to neocortex: dependence on Dlx genes. Science 278:474-476.

Ang ES, Haydar TF, Gluncic V, Rakic P (2003) Four-dimensional migratory coordinates of GABAergic interneurons in the developing mouse cortex. J Neurosci 23:5805-5815.

Ascoli GA, Alonso-Nanclares L, Anderson SA, Barrionuevo G, BenavidesPiccione R, Burkhalter A, Buzsáki G, Cauli B, Defelipe J, Fairén A, Feldmeyer D, Fishell G, Fregnac Y, Freund TF, Gardner D, Gardner EP, Goldberg JH, Helmstaedter M, Hestrin S, Karube F, et al. (2008) Petilla terminology: nomenclature of features of GABAergic interneurons of the cerebral cortex. Nat Rev Neurosci 9:557-568.

Ben-Ari Y (2002) Excitatory actions of GABA during development: the nature of the nurture. Nat Rev Neurosci 3:728-739.

Bortone D, Polleux F (2009) KCC2 expression promotes the termination of cortical interneuron migration in a voltage-sensitive calcium-dependent manner. Neuron 62:53-71.

Cavanagh ME, Parnavelas JG (1988) Development of somatostatin immunoreactive neurons in the rat occipital cortex: a combined immunocytochemical-autoradiographic study. J Comp Neurol 268:1-12.

Cowan WM, Fawcett JW, O'Leary DD, Stanfield BB (1984) Regressive events in neurogenesis. Science 225:1258-1265.

Cuzon VC, Yeh PW, Cheng Q, Yeh HH (2006) Ambient GABA promotes cortical entry of tangentially migrating cells derived from the medial ganglionic eminence. Cereb Cortex 16:1377-1388.

De Marco Garcia NV, Karayannis T, Fishell G (2011) Neuronal activity is required for the development of specific cortical interneuron subtypes. Nature 472:351-355.

Esclapez M, Tillakaratne NJ, Tobin AJ, Houser CR (1993) Comparative localization of mRNAs encoding two forms of glutamic acid decarboxylase with nonradioactive in situ hybridization methods. J Comp Neurol 331:339-362.

Esclapez M, Tillakaratne NJ, Kaufman DL, Tobin AJ, Houser CR (1994) Comparative localization of two forms of glutamic acid decarboxylase and their mRNAs in rat brain supports the concept of functional differences between the forms. J Neurosci 14:1834-1855

Fairén A, Cobas A, Fonseca M (1986) Times of generation of glutamic acid decarboxylase immunoreactive neurons in mouse somatosensory cortex. J Comp Neurol 251:67-83.

Ferreira A, Caceres A (1992) Expression of the class III beta-tubulin isotype in developing neurons in culture. J Neurosci Res 32:516-529.

Ferreira A, Busciglio J, Cáceres A (1987) An immunocytochemical analysis of the ontogeny of the microtubule-associated proteins MAP-2 and Tau in the nervous system of the rat. Brain Res 431:9-31.

Flames N, Long JE, Garratt AN, Fischer TM, Gassmann M, Birchmeier C, Lai C, Rubenstein JLR, Marín O (2004) Short- and long-range attraction of cortical GABAergic interneurons by neuregulin-1. Neuron 44:251-261.

Ganguly K, Schinder AF, Wong ST, Poo M (2001) GABA itself promotes the developmental switch of neuronal GABAergic responses from excitation to inhibition. Cell 105:521-532.

Hendry SH, Schwark HD, Jones EG, Yan J (1987) Numbers and proportions 
of GABA-immunoreactive neurons in different areas of monkey cerebral cortex. J Neurosci 7:1503-1519.

Huang ZJ, Di Cristo G, Ango F (2007) Development of GABA innervation in the cerebral and cerebellar cortices. Nat Rev Neurosci 8:673-686.

Kriegstein AR, Noctor SC (2004) Patterns of neuronal migration in the embryonic cortex. Trends Neurosci 27:392-399.

Kriegstein AR, Pitkänen A (2009) Commentary: the prospect of cell-based therapy for epilepsy. Neurotherapeutics 6:295-299.

Kuan CY, Roth KA, Flavell RA, Rakic P (2000) Mechanisms of programmed cell death in the developing brain. Trends Neurosci 23:291-297.

Lander AD, Gokoffski KK, Wan FYM, Nie Q, Calof AL (2009) Cell lineages and the logic of proliferative control. PLoS Biol 7:e15.

Lavdas AA, Grigoriou M, Pachnis V, Parnavelas JG (1999) The medial ganglionic eminence gives rise to a population of early neurons in the developing cerebral cortex. J Neurosci 19:7881-7888.

Li H, Chou SJ, Hamasaki T, Perez-Garcia CG and O'Leary DDM (2012) Neuregulin repellent signaling via ErbB4 restricts GABAergic interneurons to migratory paths from ganglionic eminence to cortical destinations. Neural Dev 7:10.

Lodato S, Rouaux C, Quast KB, Jantrachotechatchawan C, Studer M, Hensch TK, Arlotta P (2011) Excitatory projection neuron subtypes control the distribution of local inhibitory interneurons in the cerebral cortex. Neuron 69:763-779.

López-Bendito G, Sánchez-Alcañiz JA, Pla R, Borrell V, Picó E, Valdeolmillos M, Marín O (2008) Chemokine signaling controls intracortical migration and final distribution of GABAergic interneurons. J Neurosci 28:1613-1624.

Marín O, Valiente M, Ge X, Tsai L-H (2010) Guiding neuronal cell migrations. Cold Spring Harb Perspect Biol 2:a001834.

Markram H, Toledo-Rodriguez M, Wang Y, Gupta A, Silberberg G, Wu C (2004) Interneurons of the neocortical inhibitory system. Nat Rev Neurosci 5:793-807.

Menezes JR, Luskin MB (1994) Expression of neuron-specific tubulin defines a novel population in the proliferative layers of the developing telencephalon. J Neurosci 14:5399-5416.

Micheva KD, Busse B, Weiler NC, O’Rourke N, Smith SJ (2010) Singlesynapse analysis of a diverse synapse population: proteomic imaging methods and markers. Neuron 68:639-653.
Mullen RJ, Buck CR, Smith AM (1992) NeuN, a neuronal specific nuclear protein in vertebrates. Development 116:201-211.

Nadarajah B, Alifragis P, Wong ROL, Parnavelas JG (2002) Ventricledirected migration in the developing cerebral cortex. Nat Neurosci 5:218-224.

Nery S, Fishell G, Corbin JG (2002) The caudal ganglionic eminence is a source of distinct cortical and subcortical cell populations. Nat Neurosci 5:1279-1287.

O'Leary DDM, Chou S-J, Sahara S (2007) Area patterning of the mammalian cortex. Neuron 56:252-269.

Rymar VV, Sadikot AF (2007) Laminar fate of cortical GABAergic interneurons is dependent on both birthdate and phenotype. J Comp Neurol 501:369-380.

Sahara S, O'Leary DDM (2009) Fgf10 regulates transition period of cortical stem cell differentiation to radial glia controlling generation of neurons and basal progenitors. Neuron 63:48-62.

Shinozaki K, Miyagi T, Yoshida M, Miyata T, Ogawa M, Aizawa S, Suda Y (2002) Absence of Cajal-Retzius cells and subplate neurons associated with defects of tangential cell migration from ganglionic eminence in Emx1/2 double mutant cerebral cortex. Development 129:3479-3492.

Southwell DG, Froemke RC, Alvarez-Buylla A, Stryker MP, Gandhi SP (2010) Cortical plasticity induced by inhibitory neuron transplantation. Science 327:1145-1148.

Tamamaki N, Yanagawa Y, Tomioka R, Miyazaki J-I, Obata K, Kaneko T (2003) Green fluorescent protein expression and colocalization with calretinin, parvalbumin, and somatostatin in the GAD67-GFP knock-in mouse. J Comp Neurol 467:60-79.

Tanaka DH, Maekawa K, Yanagawa Y, Obata K, Murakami F (2006) Multidirectional and multizonal tangential migration of GABAergic interneurons in the developing cerebral cortex. Development 133:2167-2176.

Valcanis H, Tan S-S (2003) Layer specification of transplanted interneurons in developing mouse neocortex. J Neurosci 23:5113-5122.

Xu Q, Cobos I, De La Cruz E, Rubenstein JL, Anderson SA (2004) Origins of cortical interneuron subtypes. J Neurosci 24:2612-2622.

Yozu M, Tabata H, Nakajima K (2004) Birth-date dependent alignment of GABAergic neurons occurs in a different pattern from that of nonGABAergic neurons in the developing mouse visual cortex. Neurosci Res 49:395-403. 